\title{
The Fcp1-Wee1-Cdk1 axis affects spindle assembly checkpoint robustness and sensitivity to antimicrotubule cancer drugs
}

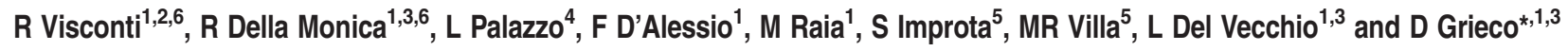

To grant faithful chromosome segregation, the spindle assembly checkpoint (SAC) delays mitosis exit until mitotic spindle assembly. An exceedingly prolonged mitosis, however, promotes cell death and by this means antimicrotubule cancer drugs (AMCDs), that impair spindle assembly, are believed to kill cancer cells. Despite malformed spindles, cancer cells can, however, slip through SAC, exit mitosis prematurely and resist killing. We show here that the Fcp1 phosphatase and Wee1, the cyclin B-dependent kinase (cdk) 1 inhibitory kinase, play a role for this slippage/resistance mechanism. During AMCD-induced prolonged mitosis, Fcp1-dependent Wee1 reactivation lowered cdk1 activity, weakening SAC-dependent mitotic arrest and leading to mitosis exit and survival. Conversely, genetic or chemical Wee1 inhibition strengthened the SAC, further extended mitosis, reduced antiapoptotic protein Mcl-1 to a minimum and potentiated killing in several, AMCD-treated cancer cell lines and primary human adult lymphoblastic leukemia cells. Thus, the Fcp1-Wee1-Cdk1 (FWC) axis affects SAC robustness and AMCDs sensitivity.

Cell Death and Differentiation (2015) 22, 1551-1560; doi:10.1038/cdd.2015.13; published online 6 March 2015

The spindle assembly checkpoint (SAC) delays mitosis exit to coordinate anaphase onset with spindle assembly. To this end, SAC inhibits the ubiquitin ligase Anaphase-Promoting Complex/Cyclosome (APC/C) to prevent degradation of the anaphase inhibitor securin and cyclin $B$, the major mitotic cyclin B-dependent kinase 1 (cdk1) activator, until spindle assembly. ${ }^{1}$ However, by yet poorly understood mechanisms, exceedingly prolonging mitosis translates into cell death induction. ${ }^{2-7}$ Although mechanistic details are still missing on how activation of cell death pathways is linked to mitosis duration, prolongation of mitosis appears crucial for the ability of antimicrotubule cancer drugs (AMCDs) to kill cancer cells. ${ }^{2-7}$ These drugs, targeting microtubules, impede mitotic spindle assembly and delay mitosis exit by chronically activating the SAC. Use of these drugs is limited, however, by toxicity and resistance. A major mechanism for resistance is believed to reside in the ability of cancer cells to slip through the SAC and exit mitosis prematurely despite malformed spindles, thus resisting killing by limiting mitosis duration. ${ }^{2-7}$ Under the AMCD treatment, cells either die in mitosis or exit mitosis, slipping through the SAC, without or abnormally dividing. ${ }^{2-4}$ Cells that exit mitosis either die at later stages or survive and stop dividing or proliferate, giving rise to resistance. ${ }^{2-4}$ Apart from a role for p53, what dictates cell fate is still unknown; however, it appears that the longer mitosis is protracted, the higher the chances for cell death pathway activation are.$^{2-7}$ Although SAC is not required per se for killing, ${ }^{6}$ preventing SAC adaptation should improve the efficacy of AMCD by increasing mitosis duration. ${ }^{2-7}$ Therefore, further understanding of the mechanisms by which cells override SAC may help to improve the current AMCD therapy. Several kinases are known to activate and sustain $\mathrm{SAC}$, and cdk1 itself appears to be of primary relevance. ${ }^{1,8,9} \mathrm{By}$ studying mitosis exit and SAC resolution, we recently reported a role for the Fcp1 phosphatase to bring about cdk1 inactivation. ${ }^{10,11}$ Among Fcp1 targets, we identified cyclin degradation pathway components, such as Cdc20, an APC/C co-activator, USP44, a deubiquitinating enzyme, and Wee1. ${ }^{10,11}$ Wee1 is a crucial kinase that controls the G2 phase by performing inhibitory phosphorylation of cdk1 at tyr-15 (Y15-cdk1). Wee1 is also in a feedback relationship with cdk1 itself that, in turn, can phosphorylate and inhibit Wee1 in an autoamplification loop to promote the G2-to-M phase transition. ${ }^{12}$ At mitosis exit, Fcp1 dephosphorylated Wee1 at threonine 239, a cdk1dependent inhibitory phosphorylation, to dampen down the cdk1 autoamplification loop, and Cdc20 and USP44, to promote APC/C-dependent cyclin B degradation. ${ }^{10-12}$ In this study we analysed the Fcp1 relevance in SAC adaptation and AMCD sensitivity.

\section{Results}

Fcp1 affects SAC-dependent mitotic delay. We previously observed that Fcp1 overexpression in HeLa cells induced faster slippage through SAC activated by the therapeutic

\footnotetext{
${ }^{1}$ CEINGE Biotecnologie Avanzate, Gaetano Salvatore 486, Naples 80145, Italy; ${ }^{2}$ IEOS, CNR, S. Pansini 5, Naples 80131, Italy; ${ }^{3}$ DMMBM, University of Naples "Federico II", S. Pansini 5, Naples 80131, Italy; ${ }^{4}$ Sir William Dunn School of Pathology, University of Oxford, Oxford OX1 3RE, UK and ${ }^{5}$ Division of Hematology, PO San Gennaro, ASLNA1 Centro, Naples 80136, Italy

*Corresponding author: D Grieco, DMMBM, CEINGE Biotecnologie Avanzate, University of Naples, "Federico II", S. Pansini 5, Naples 80131 , Italy. Tel: +39 81 3737862; Fax: +39 81 3737808; E-mail: domenico.grieco@unina.it

${ }^{6}$ These authors contributed equally to the work.

Abbreviations: AMCD, antimicrotubule cancer drug; Cdk, cyclin-dependent kinase; SAC, spindle assembly checkpoint; Mcl-1, myeloid-cell leukemia 1; APC/C, Anaphase-Promoting Complex/Cyclosome; Cdc, cell division cycle; USP, ubiquitin-specific protease; MPM-2, mitotic protein monoclonal antibody 2; siRNA, small interfering RNA; UTR, untranslated region; zVAD-fmk, z-Val-Ala-DL-Asp(OMe)-fluoromethylketone; RPMI, roswell park memorial institute medium; RPE, retinal pigmented epithelium; MCF-7, michigan cancer foundation-7; PBS, phosphate-buffered saline

Received 10.7.14; revised 15.1.15; accepted 19.1.15; Edited by H Ichijo; published online 06.3.15
} 
a
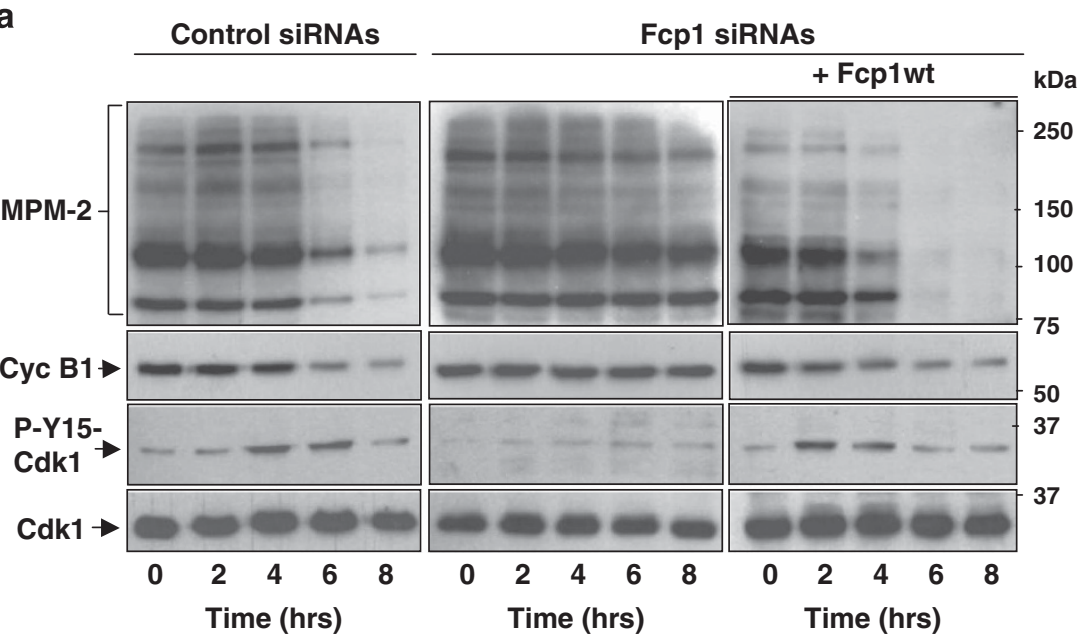

b

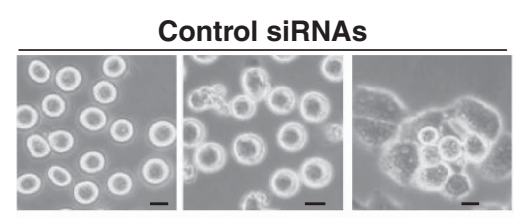

C
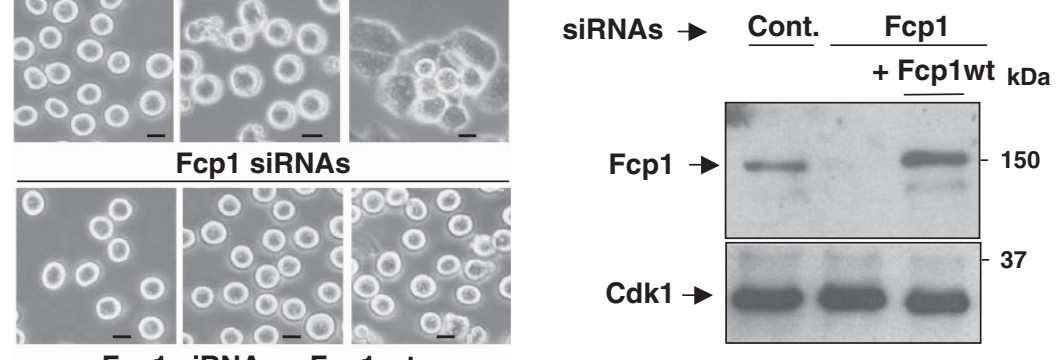
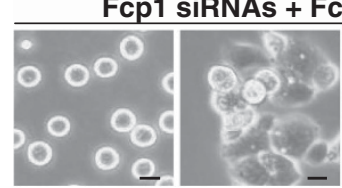

4

Time (hrs)

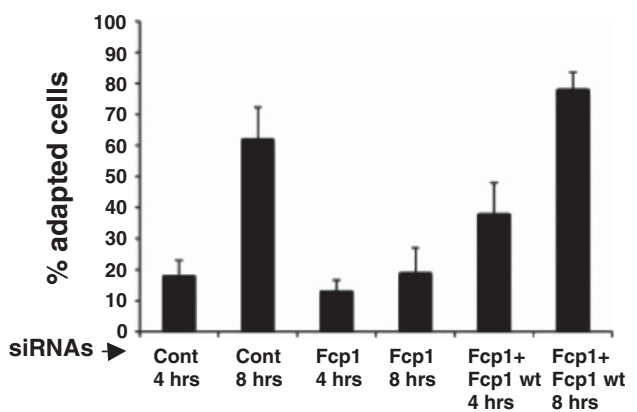

Figure 1 Fcp1 affects the duration of SAC-dependent mitotic delay. (a and $\mathbf{b})$ HeLa cells transfected with a control pool of nontargeting siRNAs (Control siRNAs), with a siRNA pool targeting the Fcp1 3'-UTR (Fcp1 siRNAs) or with Fcp1 siRNAs and a siRNA-resistant, Flag-tagged, wild-type Fcp1 (+ Fcp1wt) expression vector, were synchronized at pro-metaphase. Detached cells were collected and released into taxol-containing fresh medium (time 0 ). Cell samples were either (a) taken at indicated time points and extracts separated on SDS-PAGE, blotted and probed for the indicated antigens (MPM-2, mitotic phosphoepitopes; P-Y15-Cdk1; Cdk1; Cyc B1, cyclin B1) or (b) phase contrastphotographed at the indicated time points. Scale bars, $10 \mu \mathrm{m}$. The bar graph shows a quantitation of adapted cells expressed as the percent of input cells that reattached to the dish bottom at the indicated time points of incubation in the taxol-containing medium; error bars indicate variability within three independent experiments. (c) Extracts of control, Fcp1 siRNA-treated and Fcp1 siRNA-treated+Fcp1wt-transfected HeLa cells were probed for the indicated antigens. (d) Untransfected, pro-metaphase-synchronized HeLa cells were released into the taxol-containing fresh medium (time 0 ) and sampled at indicated time points during further incubation. Indicated antigens were directly probed on cell extract blots except for P-T239-Wee1 and Wee1 that were probed on blots of Wee1 immunoprecipitates (IPs) from the relative cell extracts. Cells were also phase contrastphotographed at the indicated time points. Scale bars, $10 \mu \mathrm{m}$. Bar graph, quantitation of adapted cells expressed as the percent of input cells that reattached to the dish bottom at the indicated time points of incubation in taxol-containing medium; error bars indicate variability within three independent experiments. Line graph, densitometric quantitation of cyclin B1 and P-T239-Wee1 signals expressed as percent of signal remaining from time 0 of incubation. (e) Fcp1 siRNA-treated and Fcp1 siRNA-treated+Fcp1wt-transfected HeLa cells, synchronized at pro-metaphase, were released into the taxol-containing fresh medium (time 0 ) and sampled at indicated time points during further incubation. Indicated antigens were probed on blots of Wee1 IPs from the relative cell extracts. The data shown are representative of at least three independent experiments 
d

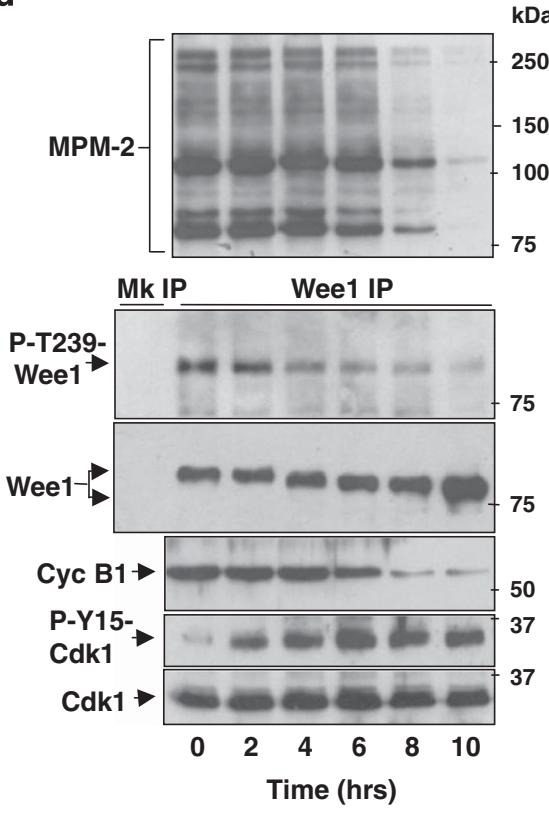

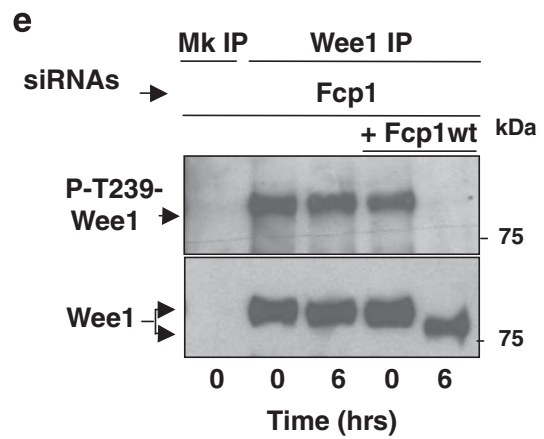

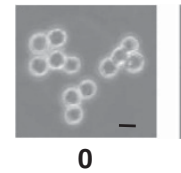
Time (hrs)

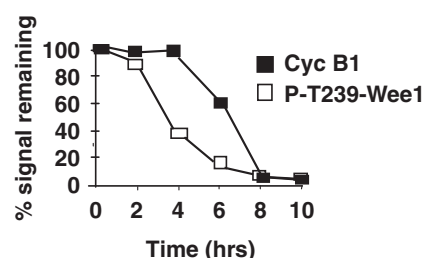

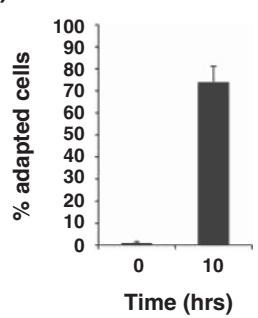

Time (hrs)

Figure 1 Continued

AMCD taxol. ${ }^{10}$ To better define how Fcp1 impinged on SAC, we asked whether genetically downregulating Fcp1 expression would affect SAC slippage. To this end, HeLa cells were treated with a control pool of nontargeting small interfering RNAs (siRNAs) or with a siRNA pool targeting the Fcp1 3'untranslated region (UTR). Fcp1 siRNA-treated cells were also transfected with a siRNAs-resistant, Flag-tagged, wildtype Fcp1 (Fcp1wt) to complement Fcp1 function. Control and Fcp1 siRNA-treated, as well as Fcp1 siRNA-treated complemented with Fcp1wt expression vector, HeLa cells were first synchronized at G1, by a double thymidine block, and then at pro-metaphase, by washing out thymidine and incubating them for $10 \mathrm{~h}$ into fresh medium containing $200 \mathrm{nM}$ nocodazole, a reversible tubulin polymerization inhibitor that almost completely prevents spindle assembly at these concentrations. Detached cells were collected, washed free of nocodazole and released into a taxol-containing fresh medium. SAC adaptation was monitored by following loss of cyclin B1 and of MPM-2, a mitotic phosphorylated protein mark, signals and reattachment of multinucleated cells to the culture dish bottom in cell samples taken at indicated time points of further incubation in the taxol-containing medium (time 0; Figures 1a and b). Fcp1 expression was efficiently downregulated by the specific siRNAs, whereas the siRNAresistant expression vector restored Fcp1 expression to about twofold over the levels of control cells (Figure 1c). Fcp1 downregulation strongly delayed slippage and mitosis exit compared with control cells, as indicated by delayed loss of cyclin B1 and of MPM-2 signals and delayed reattachment of multinucleated cells on the cell culture dish bottom, whereas expression of siRNA-resistant Fcp1wt reversed these effects and even accelerated mitosis exit kinetics compared with control cells (Figures 1a and b). Thus, Fcp1 appeared to positively affect SAC slippage. As Fcp1 controls both cyclin degradation and reactivation of the cdk1 inhibitory kinase Wee1 during mitosis exit, ${ }^{10}$ we also determined the Y15-cdk1 phosphorylation status during SAC adaptation in control siRNAs, Fcp1 siRNAs and in Fcp1 siRNAs+siRNAresistant Fcp1-treated cells. In control cells P-Y15-cdk1 increased before significant cyclin B1 degradation (Figure 1a; 4h). Fcp1 downregulation repressed P-Y15cdk1 for the duration of the experiment, whereas Fcp1 
a
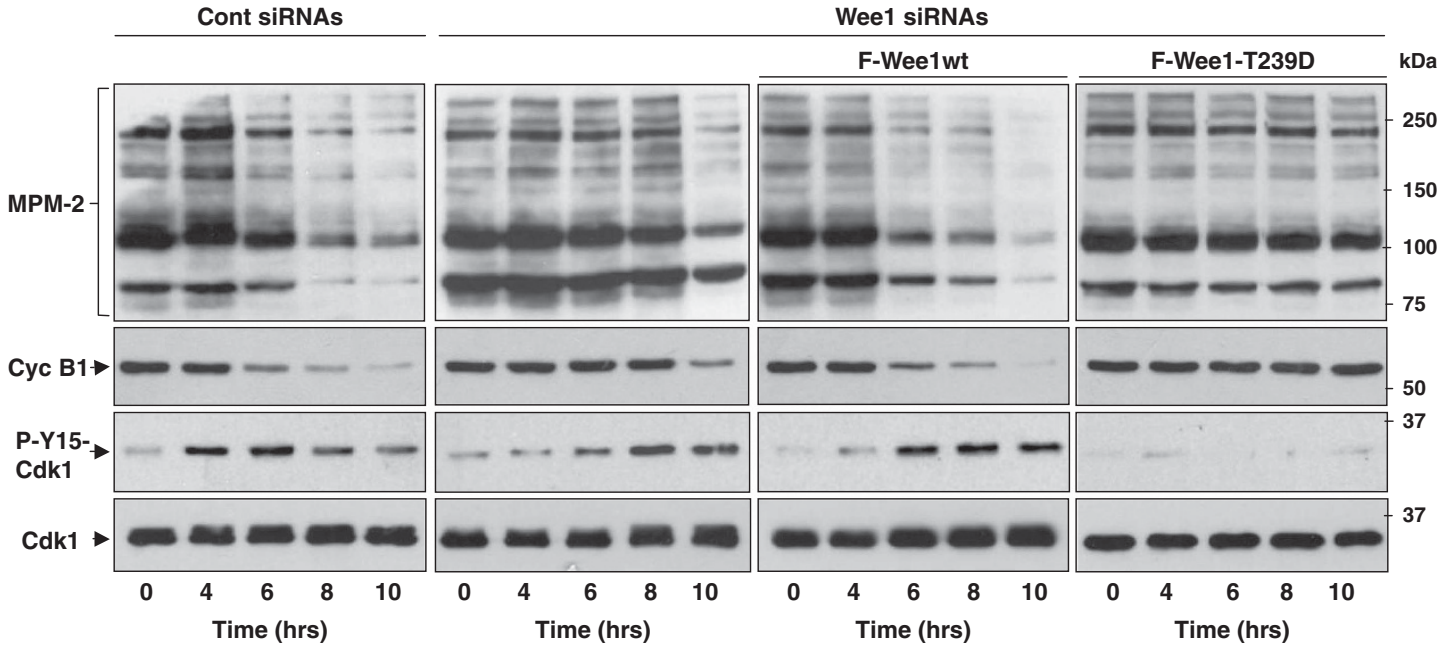

b

c

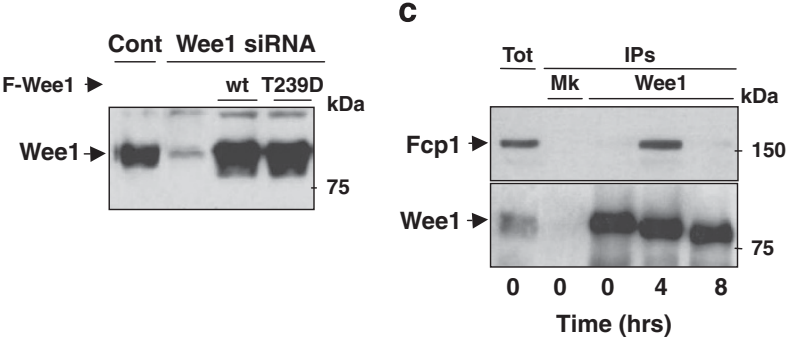

d

Wee1 siRNA
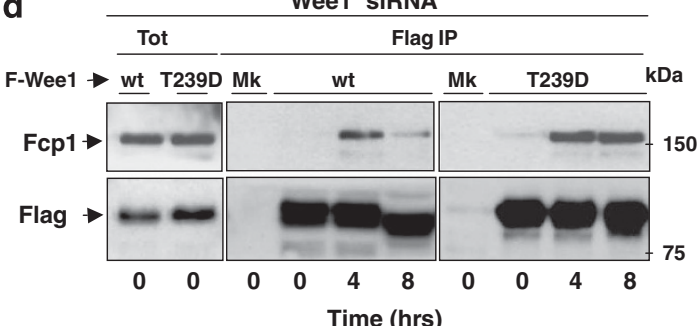

e
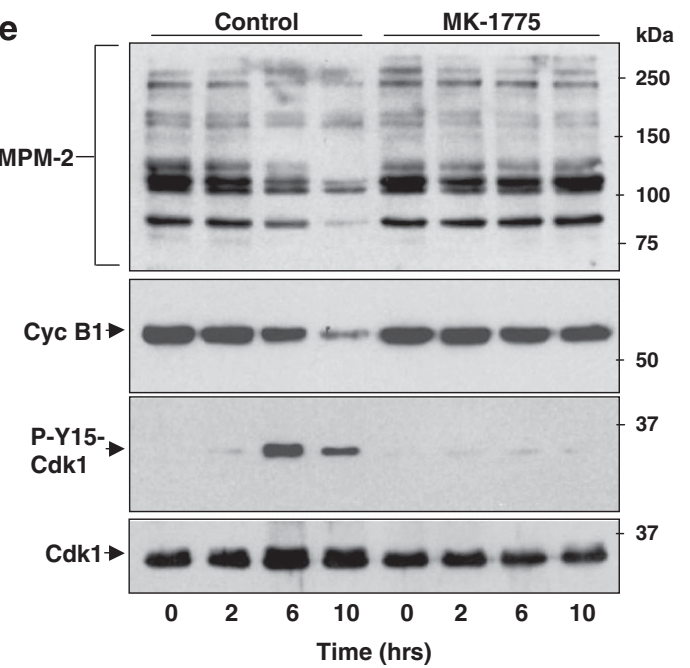

f

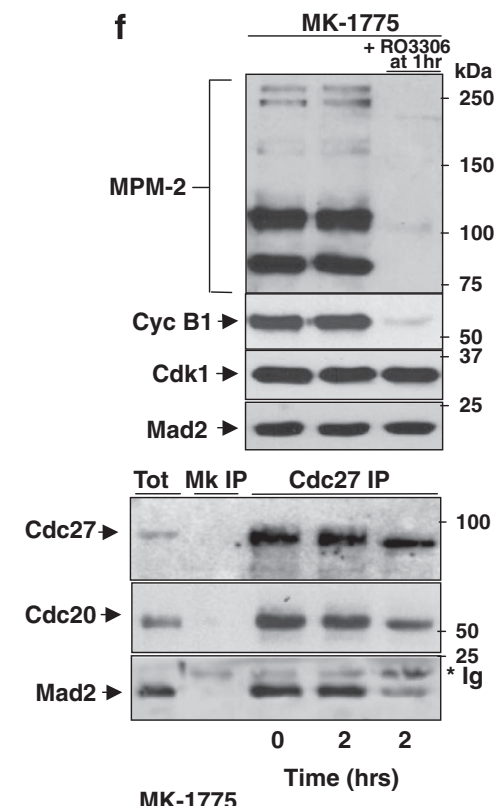

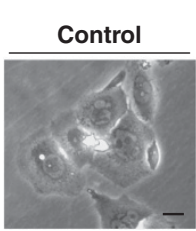

10

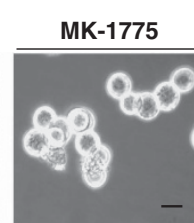

10

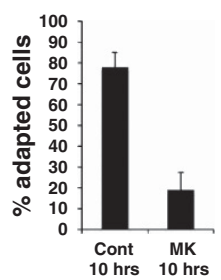

Time (hrs)
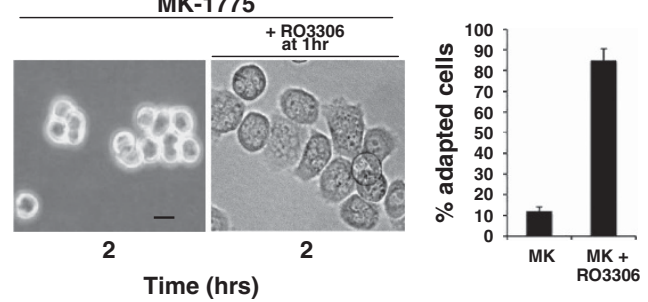
complementation reversed and accelerated $\mathrm{P}-\mathrm{Y} 15-\mathrm{cdk} 1$ appearance, as it did for cyclin B1 degradation (Figure 1a; $2 \mathrm{~h})$. Fcp1 may primarily affect cyclin B degradation and, as a consequence, Wee1 reactivation and $\mathrm{P}-\mathrm{Y} 15-\mathrm{cdk} 1$ reversal. Indeed, cyclin $B$ degradation may favor Wee1 reactivation and $P-Y 15-c d k 1$ appearance by reducing cdk1 activity. ${ }^{1,12}$ Alternatively, Fcp1 may directly dephosphorylate and stimulate Wee1 activity toward cdk1 during early slippage stages when cyclin B is still stable. In agreement with the latter hypothesis, loss of inhibitory Wee1 phosphorylation at T239, and concomitant P-Y15-cdk1 increase, begun ahead of significant cyclin B1 degradation during slippage and was tightly dependent on Fcp1 (Figures 1d and e).

The Fcp1-Wee1-Cdk1 axis affects SAC robustness. To establish a cause/effect relationship between Fcp1-dependent Wee1 reactivation and mitotic slippage, we asked whether genetic or chemical Wee1 downregulation affected SAC adaptation. To genetically downregulate Wee1, HeLa cells were treated with a pool of siRNAs targeting Wee1 3'-UTR. Control and Wee1 siRNA-treated cells were synchronized at pro-metaphase, released into the taxol-containing fresh medium (time 0) and sampled at indicated time points (Figure 2a). P-Y15-cdk1 and SAC adaptation kinetics were significantly delayed in Wee1 siRNA-treated cells (Figure 2a). In addition, expression of a siRNA-resistant, Flag-tagged, wild-type Wee1 (F-Wee1wt) reversed the delay (Figure 2a). However, expression of a mutant Wee1 version in Wee1 siRNA-treated cells bearing the substitution of threonine 239 into aspartate (FWee1-T239D) to mimic a constitutively phosphorylated residue, thus resistant to Fcp1 dephosphorylation, not only did not reverse the delay but also further extended mitosis, preventing P-Y15-cdk1 and slippage for the experiment duration (Figure 2a). Our siRNA procedure depleted $\sim 90 \%$ of Wee1 (Figure 2b). In addition, we found that Fcp1 transiently interacted with endogenous as well as exogenous Wee1 during SAC slippage (Figures $2 \mathrm{c}$ and d); thus, we interpreted these data as indicating that F-Wee1-T239D, resistant to Fcp1, acted in a dominant-negative manner by competing it out possible residual endogenous Wee1 for binding to Fcp1, sparing it from dephosphorylation and reactivation. Together, these data strongly suggest that Fcp1-dependent Wee1 reactivation promotes cdk1 inhibition via Y15-cdk1 phosphorylation that ultimately stimulates SAC slippage. We also assessed the effects of chemical Wee1 inhibition. Prometaphase-synchronized HeLa cells were released into taxolcontaining fresh medium in the absence (control) or presence of MK-1775, potent Wee1 chemical inhibitor ${ }^{13}$ and sampled at indicated time points (Figure 2e). MK-1775 treatment blocked P-Y15-cdk1 and significantly delayed SAC adaptation and mitosis exit relatively to control cells (Figure $2 e$ ). To ensure that MK-1775 was not inhibiting proteolysis or other SAC adaptation steps per se, but rather sustaining SAC robustness by preventing cdk1 inhibition by $\mathrm{Y} 15$-cdk1 phosphorylation, ${ }^{8,9}$ we asked whether chemical inhibition of cdk1, thus downstream Wee1, was able to override the MK-1775 effects. Indeed, addition of the cdk1 inhibitor RO-3306 to taxol+MK-1775treated cells led to complete and rapid SAC override as indicated by MPM-2 signal loss, cyclin B1 degradation, SAC effector protein Mad2 dissociation from $\mathrm{APC} / \mathrm{C}$ and multinucleated cells flattening on culture dish bottom (Figure 2f). Thus, the FWC axis is a critical determinant of mitosis duration under the AMCD treatment. Cdk1 activity is required, directly or indirectly, at various levels in SAC control, from kinetochore recruitment and activation of SAC proteins to their inhibitory effects on APC/C. ${ }^{1,9,10,14,15}$ In any case, sustaining cdk1 activity under SAC conditions should delay possible dissociation of SAC inhibitors from APC/C. Indeed, addition of MK-1775 significantly delayed Mad2 dissociation from APC/C observed during slippage (Supplementary Figure 1). Wee1 inhibition was able to delay slippage even in taxol-treated ovarian cancer cell line A2780 and nontransformed human hTERT-RPE1 cells as well as when SAC was activated by other AMCDs (Supplementary Figures 2 and 3). In addition, P-T239Wee1 underwent dephosphorylation in HeLa cells treated with low concentrations of nocodazole $(20 \mathrm{nM})$ that allow assembly of rudimental spindles and slippage (Supplementary Figure 4). However, Wee1 remained T239-phosphorylated in HeLa cells treated with high nocodazole concentrations $(200 \mathrm{nM})$ that did not slip through mitosis nor assembled rudimentary spindle for the duration of the experiment, suggesting that rudimentary spindle structures are required for Fcp1-dependent Wee1 dephosphorylation and reactivation (Supplementary Figure 4).

Wee1 inhibition enhances $\mathrm{Mcl}-1$ protein degradation, caspase activity and cell death under AMCDs. Protracted high colk1 activity has been mechanistically linked to cell death

Figure 2 Effects of the Fcp1-Wee1-Cdk1 axis on SAC-dependent mitotic delay. (a) Prior to be pro-metaphase-synchronized, HeLa cells were treated with either nontargeting siRNA (Cont siRNAs) or with siRNAs targeting Wee1 3'-UTR (Wee1 siRNAs). Wee1 siRNA-treated cells were also complemented with 3XFlag-tagged Wee1 wild-type or T239D mutant (F-Wee1wt and F-Wee-T239D) expression vectors. Pro-metaphase-synchronized cells were released into taxol-containing fresh medium (time 0), sample taken at indicated time points and extracts probed for indicated antigens. (b) Wee1 expression levels in cells treated as described in a. (c) Pro-metaphase-synchronized HeLa cells were released into the taxol-containing fresh medium and taken at indicated time points. Mock or Wee1 IPs (Mk; Wee1) were performed from cell extracts and probed for indicated antigens. (d) Wee1 siRNA-treated HeLa cells transfected with F-Wee1wt or F-Wee1-T239D expression vectors or an empty vector (Mk) were pro-metaphase-synchronized, released into the taxol-containing fresh medium (time 0 ) and sampled at indicated time points. Anti-Flag IPs were performed from cell extracts and probed for indicated antigens. (e) Pro-metaphase-synchronized HeLa cells were released into the taxol-containing fresh medium (time 0 ) in the absence (Control) or presence of the Wee1 inhibitor MK-1775. Cell samples were either taken at indicated time points of incubation and extracts probed for indicated antigens or photographed in phase contrast at indicated time points. Scale bars, $10 \mu \mathrm{m}$. Bar graph: quantitation of adapted cells expressed as the percent of input cells that reattached to the dish bottom at the indicated time points of incubation in the taxol-containing medium; error bars indicate variability within three independent experiments. (f) Pro-metaphase-synchronized HeLa cells were released into taxol- and MK-1775containing fresh medium and split into two portions. After 1-h incubation, one portion received the cdk1 inhibitor R0-3306, and incubation was prolonged for another hour for all portions. Cell extracts were directly probed for indicated antigens or processed for Cdc27 IPs that were subsequently probed for Cdc27, Cdc20 and Mad2 (Mk IP; mock IPs). Cells were also photographed in phase contrast at indicated time points. Bar graph: quantitation of adapted cells expressed as the percent of input cells that reattached to the dish bottom at the indicated time points of incubation in the taxol-containing medium; error bars indicate variability within three independent experiments. Scale bars, $10 \mu \mathrm{m}$. The data shown are representative of at least three independent experiments 
through activation of various death pathways. ${ }^{2}$ In particular, the antiapoptotic protein $\mathrm{Mcl}-1$ undergoes degradation in mitosis in a cdk1-dependent manner and this appears crucial for AMCDinduced apoptosis. ${ }^{2,7}$ Analysis of Mcl-1 levels, from samples of the experiment described in Figure 2a, showed that the low protein concentration in control-siRNA-treated mitotic cells increased as the cells slipped through mitosis, whereas in Wee1-siRNA-treated cells the Mcl-1 level was further reduced during prolonged mitosis, although it still appeared to increase again as cells finally attempted to exit mitosis (Figure 3a). Complementation with F-Wee1wt restored Mcl-1 accumulation kinetics as in control cells, whereas F-Wee1-T239D expression, which blocked mitosis exit for the experiment duration, reduced $\mathrm{Mcl}-1$ to almost undetectable levels (Figure 3a). To determine whether Wee1 affected cell death and apoptosis in addition to SAC slippage, we assayed cell viability, by trypan blue

a
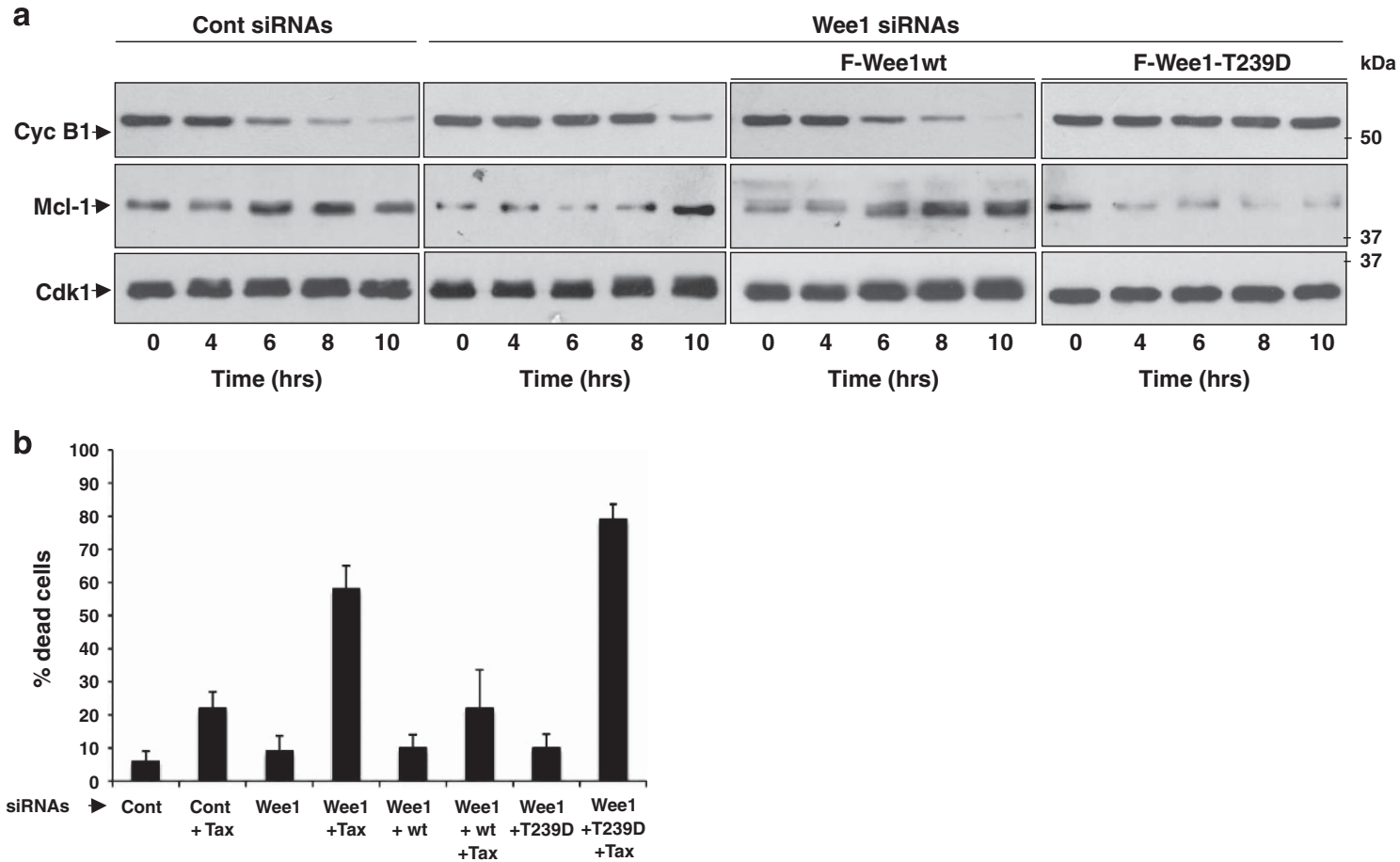

C
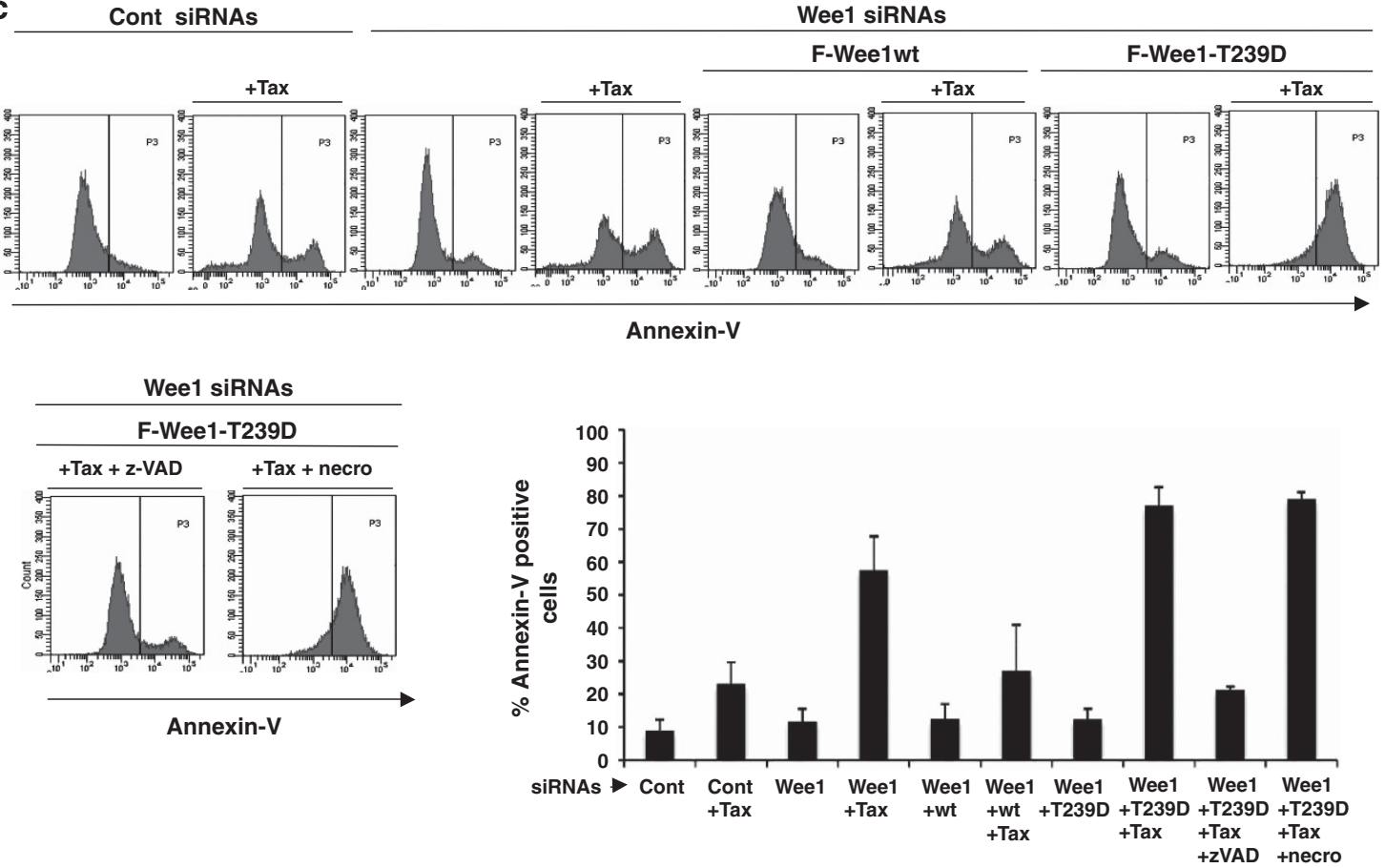

Figure 3 Continued 
d
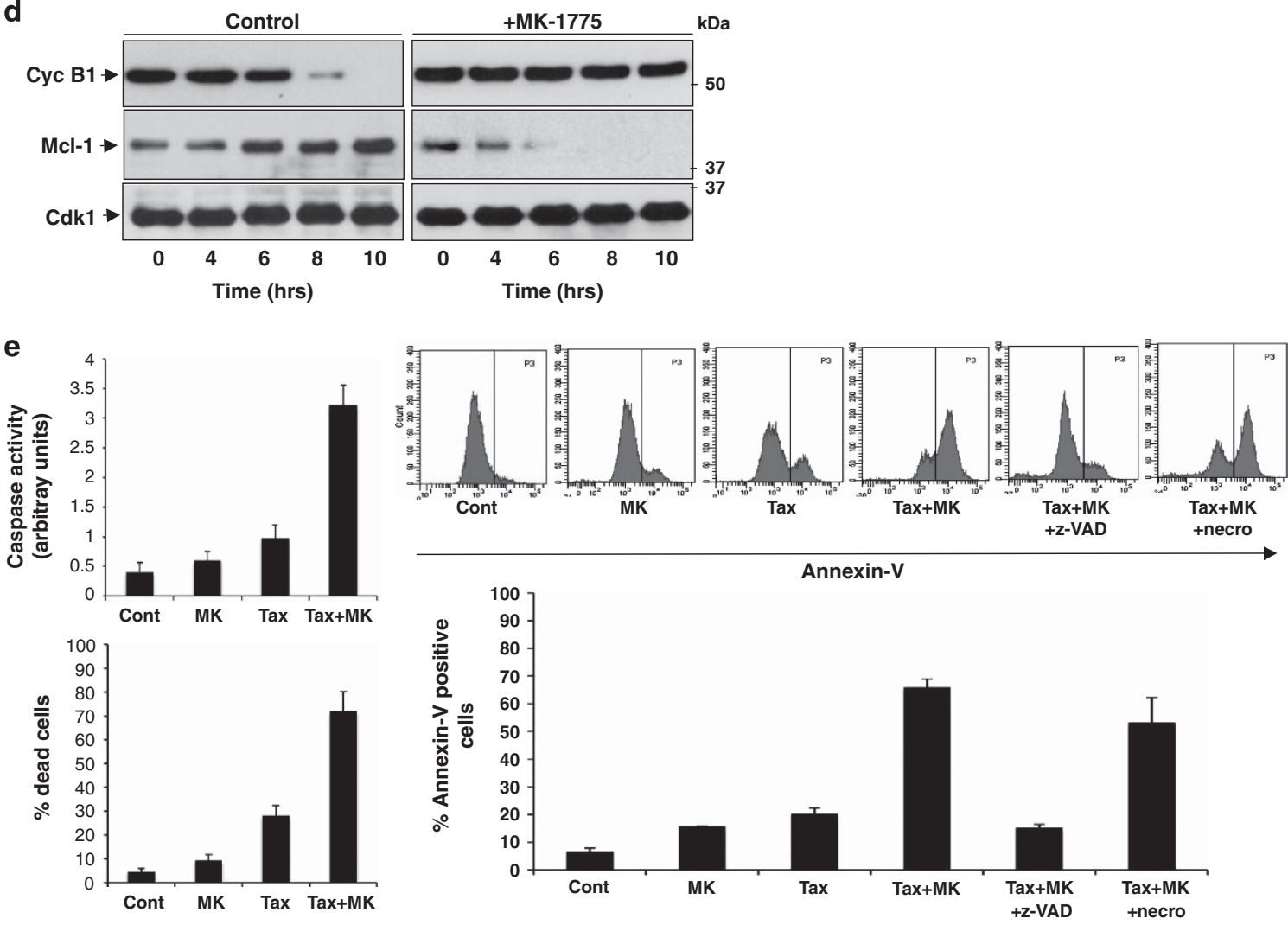

Figure 3 Wee1 downregulation enhances degradation of the antiapoptotic Mcl-1 protein, caspase activity and cell death under taxol regimen. (a) The same cell extracts of the experiment described in Figure 2a were probed for the Mcl-1 protein (to follow SAC adaptation kinetics, Cyc B1 and Cdk1 were also reported from the experiment described in Figure 2a). ( $\mathbf{b}$ and $\mathbf{c}$ ) HeLa cells were treated as described in Figure 2. Then, $36 \mathrm{~h}$ post pro-metaphase release, either (b) cell death was measured by trypan blue exclusion and expressed in the bar graph as percent of total cells (error bars indicate variability within three independent experiments) or (c) Annexin-V positivity was scored by flow cytometry. In c, panels of flow cytometry analysis for Annexin- $V$ staining of cell samples from a representative experiment. Samples of Wee1 siRNA-treated and reconstituted with mutant F-Wee1-T239D cells were also incubated with the caspase inhibitor Z-VAD-fmk or the necroapoptosis inhibitor necrostatin-1 from the time of taxol addition (Wee1 siRNA-F-Wee1T239D +Tax+z-VAD and +Tax+necro, respectively). The bar graph shows a quantitation of Annexin-V positivity expressed as percent of flow cytometry-analysed cells (error bars indicate variability within three independent experiments). The cutoff to count for Annexin-V positivity was established by imposing P3 marker between the end of the bell-shaped part and the brighter tail part of Annexin-V signal distribution histograms from control siRNA-treated cell populations incubated in the absence of taxol. (d) HeLa cells were treated as described in Figure 2e. Mcl-1, cyclin B1 (Cyc B1) and cdk1 were probed from extracts of cell samples taken at the indicated time points. (e) Pro-metaphase-synchronized HeLa cells were released just into fresh medium (Control), into fresh medium supplemented with MK-1775 (MK), into taxol-containing fresh medium (Tax) or into taxol-containing fresh medium supplemented with MK-1775 (Tax+MK). Left top graph, caspase activity was determined from cell samples $24 \mathrm{~h}$ post pro-metaphase release. Left bottom graph, cell death was measured $36 \mathrm{~h}$ post pro-metaphase release by trypan blue exclusion and expressed as percent of total cells. Right panels, flow cytometry analysis panels of Annexin-V staining of cell samples measured $36 \mathrm{~h}$ post pro-metaphase release from a representative experiment. Cell samples treated with taxol and MK-1775 (Tax+MK) were also incubated with z-VAD-fmk (+z-VAD) or necrostatin-1 (+necro) from the time of Tax+MK addition. The cutoff to count for Annexin-V positivity was established by imposing P3 marker between the end of the bell-shaped part and the brighter tail part of Annexin-V signal distribution histograms of control cell populations incubated in the absence of other treatments post-prometaphase release. Right bottom graph, quantitation of Annexin-V positivity expressed as percent of flow cytometry-analysed cells. Error bars indicate variability within three independent experiments

exclusion, and positivity for the apoptotic marker Annexin-V, by flow cytometry, $36 \mathrm{~h}$ post pro-metaphase release in cells treated as described in Figure 2a (Figures $3 b$ and c). Compared with control cells, Wee1-siRNA-treated cells appeared significantly more sensitive to the taxol treatment (Figures $3 b$ and $c$ ). In these cells, expression of F-Wee1wt reduced sensitivity to levels comparable to control cells, whereas expression of the F-Wee1-T239D mutant induced highest sensitivity to taxol (Figures $3 \mathrm{~b}$ and $\mathrm{c}$ ). In addition, Annexin- $\mathrm{V}$ positivity was also strongly reduced in the F-Wee1-T239D mutant-expressing cells treated with the caspase inhibitor Z-VAD-fmk from the time of taxol addition, whereas treatment with the necroapoptosis inhibitor necrostatin-1, from the same time, had minor effects (Figure 3c). A similar increase in Mcl-1 destabilization and taxol sensitivity could be observed upon Fcp1 downregulation in HeLa cells (Supplementary Figure 5). We also assessed Mcl-1 stability, caspase activity in taxol-treated HeLa cells upon chemical Wee1 inhibition by MK-1775 (Figures 3d and e). Strong Mcl-1 protein downregulation was evident during MK-1775-dependent prolonged mitotic arrest in taxol-treated HeLa cells (Figure 3d). MK-1775 and taxol combination resulted also in substantial potentiation of caspase activition and apoptotic cell death compared with single treatments (Figure 3e). MK-1775 and taxol combination sustained the mitotic state, as indicated by maintenance of MPM-2 signals, and increased cell death also when added to asynchronous populations and at therapeutic doses in HeLa cells (Figure 4a and Supplementary Figure 6a). AMCDs are used for the 
therapy of a variety of cancer types; for instance, vincristine is a major drug in lymphoblastic leukemia treatment. ${ }^{16}$ When combined with vincristine, MK-1775 potently sustained maintenance of MPM-2 signals, indicating sustained mitotic state, and increased apoptotic cell death at later times in asynchro- nously growing TOM-1 and MOLT-4 cells, stabilized B and T lymphoblastic leukemia cells, respectively (Figures $4 \mathrm{~b}$ and $\mathrm{c}$ and Supplementary Figures $6 b$ and c). Increased sensitivity to taxol was also induced by combined treatment with MK-1775 in asynchronous cell populations of A2780 and MCF-7, a breast a

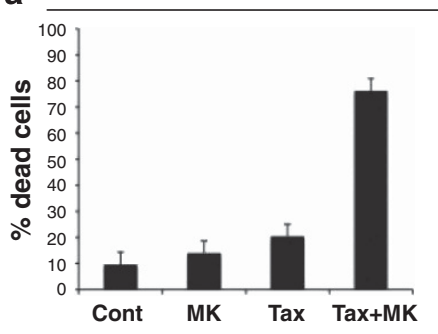

b

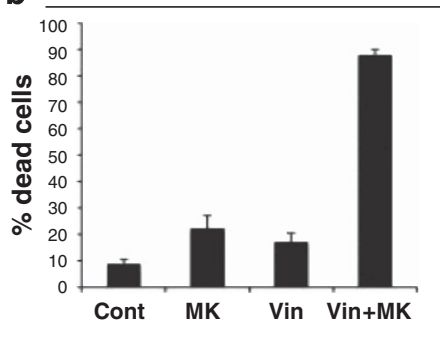

C

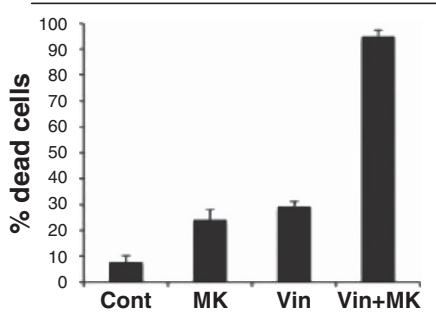

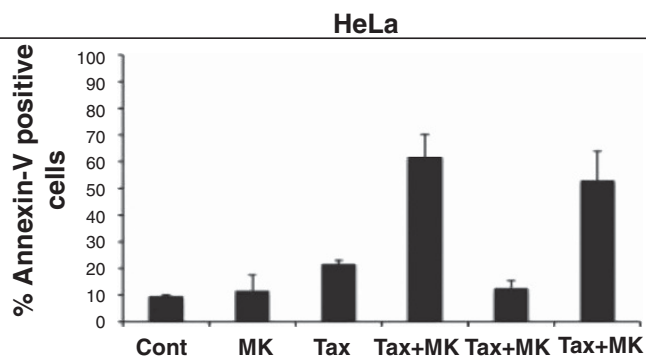

$+z$-VAD +necro

TOM-1

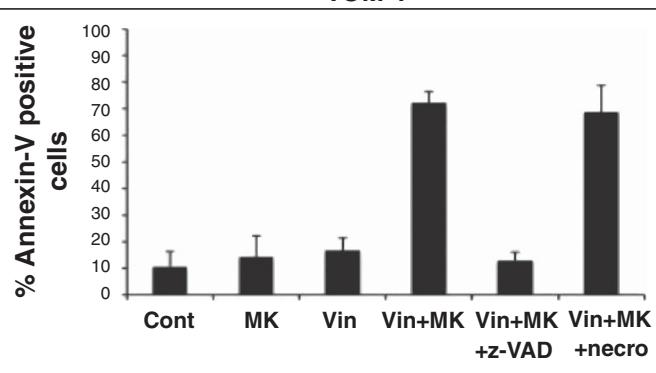

MOLT-4

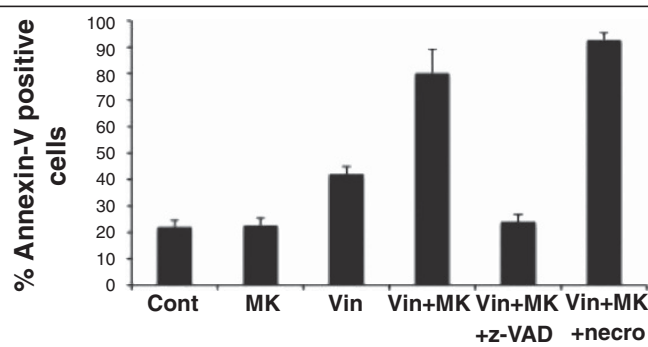

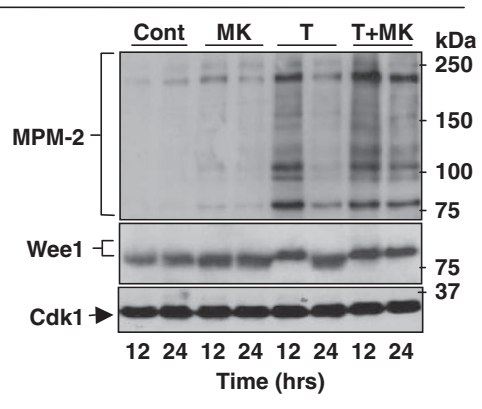

Cont MK Vin Vin+MK kDa

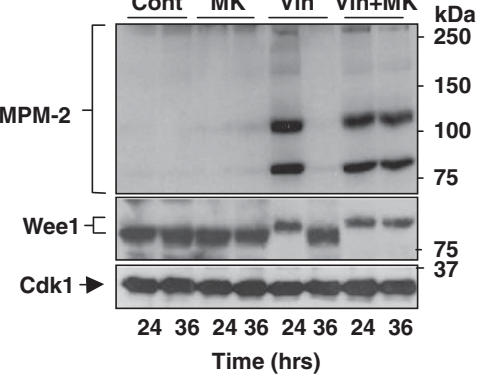

d

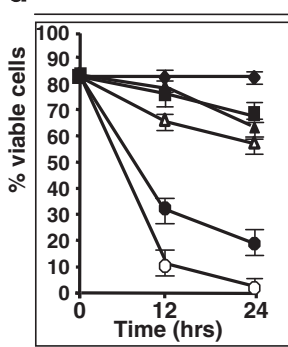

Patient 1 Pro B ALL (95 \% blasts)

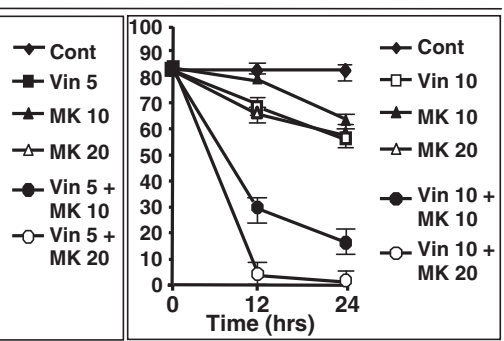

Patient 3 Pre B ALL (75 \% blasts)

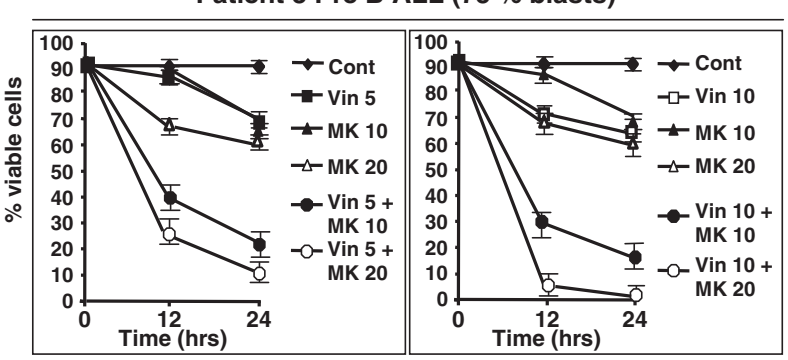

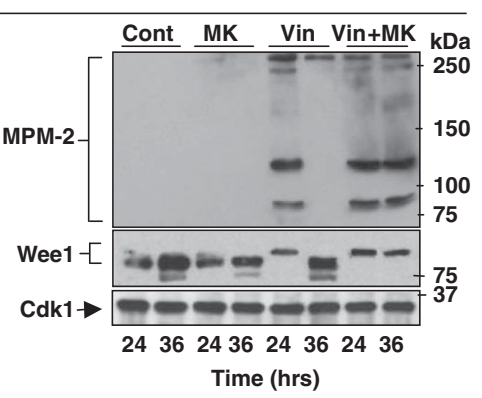

Patient 2 Pro B ALL (92 \% blasts)

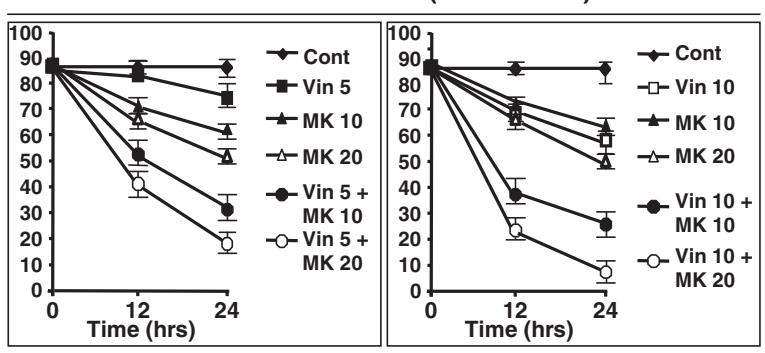

Patient 4 Pro T ALL (97 \% blasts)

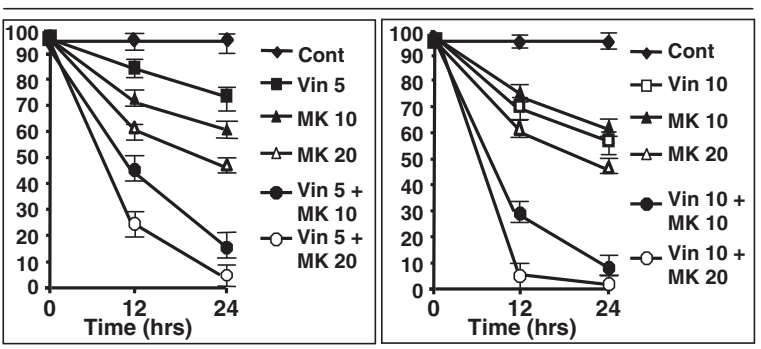


cancer cell line (Supplementary Figure 7). Similarly, increased AMCD sensitivity was induced in asynchronous HeLa, TOM-1 and MOLT-4 cells upon genetic downregulation of Wee1 or Fcp1 (Supplementary Figure 8). To further assess the possible therapeutic value of the combination of AMCDs with Wee1 inhibition, the combination of MK-1775 and vincristine was tested on primary leukemic blasts from bone marrow specimens of four adult lymphoblastic leukemia patients. Figure $4 \mathrm{e}$ shows that the combination potently synergized in reducing viability compared with single drug treatments.

\section{Discussion}

Our data show that Fcp1-dependent Wee1 reactivation and cdk1 inhibitory phosphorylation promote SAC slippage and mitosis exit in the presence of AMCDs. Perhaps, rudimentary spindle assembly is sufficient to allow the FWC axis to depress cdk1 activity to a point in which SAC is no longer able to inhibit APC/C. ${ }^{1,8,9}$ Conversely, Wee1 inhibition helps in prolonging mitosis under SAC and enhances AMCD-induced cell killing. ${ }^{2-4,6}$ Wee1 is instrumental for the DNA damage G2-to$M$ checkpoint, and Wee1 inhibitors are currently under clinical study in combination with DNA-damaging drugs in keeping with the idea that pushing DNA-damaged cancer cells into mitosis would help in their killing. ${ }^{17}$ By suggesting that Wee1 inhibitors could be beneficial in combination with AMCDs, our data may further expand our therapeutic armory against cancer. In particular, we hypothesize that the association of Wee1 inhibitors with AMCDs will be potentially beneficial in several cases in which AMCDs are used as monotherapeutic agents as, for instance, in second-line therapeutic regimens for several solid and hematological malignancies.

\section{Materials and Methods}

Cell cultures. HeLa and hTERT-RPE1 cells were grown as previously described. ${ }^{10}$ A2780, TOM-1 and MOLT-4 cells were grown in RPMI (Sigma-Aldrich, St. Louis, MO, USA) with $2 \mathrm{mM}$ L-glutamine and $10 \%$ fetal bovine serum (FBS; GE Healthcare Hyclone, Little Chalfont, Buckinghamshire, UK), MCF-7 cells in Eagle's Minimum Essential Medium (Sigma-Aldrich) with 10\% FBS, 2 mM L-glutamine and $1 \%$ nonessential amino acids. Phase contrast photographs were performed with a Leica DFC 320 microscope (Leica Microsystems GmbH, Wetzlar, Germany).

Chemicals and treatments. Pro-metaphase-synchronized cell populations were obtained in the following way: cells were treated with $4 \mathrm{mM}$ thymidine for $18 \mathrm{~h}$, released from the first thymidine block into fresh medium for $6 \mathrm{~h}$ and then treated again with thymidine for other $18 \mathrm{~h}$. Cells were released from the second thymidine block into fresh medium containing $200 \mathrm{nM}$ nocodazole for $10 \mathrm{~h}$. Detached cells were collected, washed free of nocodazole and replated under the various, described, conditions. Taxol (paclitaxel; Calbiochem, Merck Millipore, Billerica, MA, USA) was used at $25 \mathrm{nM}$ in experiments with synchronized and at $10 \mathrm{nM}$ with asynchronous HeLa and A2780 cell populations, at $10 \mathrm{nM}$ for MCF-7 cells and at $500 \mathrm{nM}$ for hTERTRPE1. In combination treatment experiments with taxol, MK-1775 (Calbiochem, Merck Millipore) was added at 500 and $200 \mathrm{nM}$ in synchronized and asynchronous cell populations, respectively, except in experiments with hTERT-RPE1 cells, which were used always at $500 \mathrm{nM}$. Vincristine (Calbiochem, Merck Millipore) was used at $25 \mathrm{nM}$ in experiments with HeLa cells and at 5 or $10 \mathrm{nM}$ in experiments with lymphoblasts as indicated. In experiments that included combination treatments of vincristine and MK-1775 in lymphoblasts, MK-1775 was used at 10 or $20 \mathrm{nM}$ as indicated. Necrostatin-1 and z-VAD-fmk (Calbiochem, Merck Millipore) were used at 50 and $25 \mathrm{nM}$, respectively. Nocodazole (Calbiochem, Merck Millipore) was used at 20 or $200 \mathrm{nM}$ as indicated and S-Trityl-L-cysteine (STLC; Calbiochem, Merck Millipore) at $10 \mu \mathrm{M}$.

Transfections, siRNAs and site-directed mutagenesis. Expression vector transfections were performed using Linear Polyethylenimine (PEl; Polysciences Inc., Warrington, PA, USA). 3XFlag-Fcp1wt and 3XFlag-Fcp1CD expression vectors were obtained as described..$^{10}$ For experiments with transfected and synchronized cells, HeLa cells were transfected $8 \mathrm{~h}$ before the synchronization procedure. siRNAs targeting the $3^{\prime}$-UTR of human WEE1 (5'-gggcuuu auuacagacauauu- $3^{\prime}, \quad 5^{\prime}$-guacauagcuguuugaaauuu- $3^{\prime}$ and $5^{\prime}$-uguaaacuuguagc aunauu-3') were purchased from Dharmacon Inc. (Lafayette, CO, USA). To generate the Wee1-T239D-mutant version, threonine 239 residue of human Wee1 was mutagenized into aspartate (5'-cgacagactcctcaagtgaatattaatcctttgatc-3') with the QuikChange II XL site-directed mutagenesis kit (Agilent Technologies, Santa Clara, CA, USA) in a 3XFlag-Wee1 expression construct as template. For siRNA treatment and complementation experiments in synchronized cells, first HeLa cells were mock- or 3XFlag-Wee1 expression construct-transfected. Eight hours post transfection, cells were treated with thymidine $(4 \mathrm{mM})$ and $18 \mathrm{~h}$ later cells were released from the first thymidine block and either transfected with nontargeting or specific siRNAs. Six hours later, cells were treated again with thymidine for further $18 \mathrm{~h}$, and then washed and incubated in fresh medium for $9 \mathrm{~h} ; 200 \mathrm{nM}$ nocodazole was added and detached cells collected after $2 \mathrm{~h}$ of further incubation. For siRNA experiments with asyncronous cells, HeLa, MOLT-4 and TOM-1 cells were transfected with nontargeting or specific siRNAs $48 \mathrm{~h}$ before treatment with the indicated drugs. Flow cytometry analysis was conducted after further incubation as indicated.

Patient specimens, clinical information and flow cytometric immunophenotyping. This study included four patients affected by acute lymphoblastic leukemia (ALL). Mononuclear cells were obtained by incubating bone marrow samples in lysing solution $\left(150 \mathrm{mM} \mathrm{NH}_{4} \mathrm{Cl}, 10 \mathrm{mM} \mathrm{KHCO}_{3}\right.$ and $1 \mathrm{mM} \mathrm{EDTA}$; 1:20 dilution) for $10 \mathrm{~min}$ at room temperature, and by washing cell pellets with Dulbecco phosphate-buffered saline (PBS; Sigma-Aldrich) supplemented with $5 \%$ FBS. Cells were, then, resuspended in RPMI supplemented with $2 \mathrm{mM} \mathrm{L-glutamine}$

\footnotetext{
Figure 4 Wee1 inhibition enhances AMCD-dependent cell death in stabilized cell cultures and in primary human adult lymphoblastic leukemia specimens. (a) Asynchronous HeLa cells were either mock-treated (Cont) or treated with MK-1775 (MK) or with taxol (Tax) or with a combination of MK-1775 and taxol (Tax+MK). Left graph, cell death was measured at $36 \mathrm{~h}$ from treatments by trypan blue exclusion and expressed as percent of total cells. Middle graph, quantitation of Annexin-V positivity expressed as percent of flow cytometry-analysed cells measured $36 \mathrm{~h}$ post-pro-metaphase release; cells were also incubated with z-VAD-fmk (+z-VAD) or necrostatin-1 (+necro) from the time of Tax+MK addition. The cutoff to count for Annexin-V positivity was established by imposing P3 marker between the end of the bell-shaped part and the brighter tail part of Annexin-V signal distribution histograms of control cell populations incubated in the absence of any treatment. Error bars indicate variability within three independent experiments. Right panels, indicated antigens were probed from cell extracts taken at the indicated time points from treatments. (b) Asynchronous TOM-1 and (c) MOLT-4 cells were either untreated (Cont) or treated with $20 \mathrm{nM}$ MK-1775 (MK) or $10 \mathrm{nM}$ vincristine (Vin) or with combinations of vincristine and MK-1775 (Vin+MK). Left graph, cell death was measured at $48 \mathrm{~h}$ from treatments by trypan blue exclusion and expressed as percent of total cells. Middle graph, quantitation of Annexin-V positivity expressed as percent of flow cytometry-analysed cells measured $48 \mathrm{~h}$ post-pro-metaphase release; cells were also incubated with z-VAD-fmk (+z-VAD) or necrostatin-1 (+necro) from the time of Vin+MK addition. The cutoff to count for Annexin-V positivity was established by imposing P3 marker between the end of the bell-shaped part and the brighter tail part of Annexin-V signal distribution histograms of control cell populations incubated in the absence of any treatment. Error bars indicate variability within three independent experiments. Right panels, indicated antigens were probed from extracts of cell samples taken at the indicated time points from the previously described treatments. (d) Percent of viability of leukemic blast specimens, deriving from bone marrow blood of four adult lymphoblastic leukemia patients (Patients 1-4), measured by trypan blue exclusion and expressed as percent of total cells at the indicated time points from the following additions: mock addition (Cont), 10 or $20 \mathrm{nM}$ MK and (left graphs) $5 \mathrm{nM}$ vincristine alone or in combination with 10 or $20 \mathrm{nM}$ MK or (right graphs) $10 \mathrm{nM}$ vincristine alone or in combination with 10 or $20 \mathrm{nM} \mathrm{MK}$. Diagnosis and percent of leukemic blasts in the bone marrow mononuclear cell sample is indicated per patient; error bars indicate variability within triplicate assays
} 
and $20 \%$ FBS. The diagnosis was carried out on bone marrow-purified mononuclear cells, according to morphological, cytometric and genotypic criteria established by World Health Organization classification. ${ }^{18}$ Patients 1,2 and 3 had overt B-ALL and patient 4 showed T-ALL. In particular, patients 1, 2 and 4 were at initial diagnosis, whereas patient 3 was at relapse after treatment according to the GIMEMA LAL0904 protocol. ${ }^{19}$ Patients' age ranged from 20 to 43 years. Written informed consent was obtained from all four patients before the study was conducted. The following antigens were studied at the bone marrow level during diagnostic procedures by six-color immunophenotyping staining: CD34, CD45, CD10, HLA-DR, CD19, CD20, CD22, CD24, CD38, CD69, TdT, CD2, CD3, CD4, CD5, CD7, CD8, CD1a, CD13, CD33 and CD117. All monoclonal antibodies were purchased from Becton Dickinson (San Jose, CA, USA) and Beckman Coulter (Miami, FL, USA).

Other immunological procedures. Anti-P-Y15-cdk1, MPM-2 and Flag antibodies were from Cell Signaling Technologies (Danvers, MA, USA), Merck Millipore (Billerica, MA, USA) and Sigma-Aldrich respectively. Anti-Fcp1 antibodies were from Bethyl Laboratories (Montgomery, TX, USA) and Santa Cruz Biotechnology (Dallas, TX, USA). Rabbit polyclonal antibodies against P-T239 of human Wee1 were previously described. ${ }^{10}$ Other antibodies were from Santa Cruz Biotechnology. Immunoprecipitations and immunoblots were performed as described. ${ }^{10}$ Densitometric quantitations of immunoblot signal intensity were performed by using the ImageJ software (National Institutes of Health, Bethesda, MD, USA).

Caspase activity and cell death determinations. For cell viability/death assays, at the end of each treatment, triplicate cell samples were stained with $0.05 \%$ trypan blue and cells excluding/including the dye were quantitated visually at the microscope (four fields per sample). For caspase activity assays, cell pellets were washed with PBS and lysed in $30 \mathrm{mM}$ Tris $\mathrm{HCl}, 150 \mathrm{mM} \mathrm{NaCl}, 10 \%$ Triton and 10\% glycerol. After 20-min incubation on ice, lysates were cleared using centrifugation at $4^{\circ} \mathrm{C}$ and assayed $(10 \mu \mathrm{g})$ by incubation at $37^{\circ} \mathrm{C}$ for $1 \mathrm{~h}$ in $100 \mu \mathrm{l}$ of AFC buffer (20 mM HEPES pH 7.5, 1\% sucrose, 0.1\% CHAPS and $10 \mathrm{mM}$ DTT) including $50 \mu \mathrm{M}$ Z DEVD-AFC (Enzyme System Products Inc., Dublin, CA, USA). Fluorescence was measured through EnVision 2102 Multilable Reader (Perkin Elmer, Inc., Waltham, MA, USA).

Flow cytometry. Annexin- $V$ staining was determined by flow cytometry using a FITC-conjugated Annexin-V Apoptosis Detection kit and a FACSCanto II flow cytometer (BD Biosciences, Becton Dickinson). The cutoff for Annexin-V positivity was established by imposing a P3 marker between the end of the bell-shaped part and the brighter tail part of the Annexin-V signal distribution histograms from control cell populations per cell type and experiment and kept unchanged for all samples of the same experiment.

\section{Conflict of Interest}

The authors declare no conflict of interest.

Acknowledgements. We wish to thank VE Avvedimento, RM Melillo and F Carlomagno for their helpful suggestions. This study was supported by Associazione Italiana per la Ricerca sul Cancro (AIRC) and Italian Ministero dell'Università e della Ricerca PRIN 2010-2011. LP is a recipient of an AIRC fellowship for abroad.
1. Musacchio A, Salmon ED. The spindle-assembly checkpoint in space and time. Nat Rev Mol Cell Biol 2007; 8: 379-393.

2. Topham CH, Taylor SS. Mitosis and apoptosis: how is the balance set? Curr Opin Cell Biol 2013; 25: 780-785

3. Swanton C, Marani M, Pardo O, Warne PH, Kelly G, Sahai E et al. Regulators of mitotic arrest and ceramide metabolism are determinants of sensitivity to paclitaxel and other chemotherapeutic drugs. Cancer Cell 2007; 11: 498-512.

4. Gascoigne KE, Taylor SS. Cancer cells display profound intra- and interline variation following prolonged exposure to antimitotic drugs. Cancer Cell 2008; 14: 111-122.

5. Rieder CL, Maiato H. Stuck in division or passing through: what happens when cells cannot satisfy the spindle assembly checkpoint. Dev Cell 2004; 7: 637-651.

6. Huang $\mathrm{H}-\mathrm{C}$, Shi J, Orth JD, Mitchison TJ. Evidence that mitotic exit is a better cancer therapeutic target than spindle assembly. Cancer Cell 2009; 16: 347-358.

7. Harley ME, Allan LA, Sanderson HS, Clarke PR. Phosphorylation of Mcl-1 by CDK1-cyclin B1 initiates its Cdc20-dependent destruction during mitotic arrest. EMBO J 2010; 29 : 2407-2420.

8. D'Angiolella V, Mari C, Nocera D, Rametti L, Grieco D. The spindle checkpoint requires cyclin-dependent kinase activity. Genes Dev 2003; 17: 2520-2525.

9. D'Angiolella V, Grieco D. Attach first, then detach: a role for cyclin B-dependent kinase 1 in coordinating proteolysis with spindle assembly. Cell Cycle 2004; 3: 132-133.

10. Visconti R, Palazzo L, Della Monica R, Grieco D. Fcp1-dependent dephosphorylation is required for M-phase-promoting factor inactivation at mitosis exit. Nat Commun 2012; 3: 894.

11. Visconti R, Palazzo L, Pepe A, Della Monica R, Grieco D. The end of mitosis from a phosphatase perspective. Cell Cycle 2013; 12: 17-19.

12. Hunt $T$. On the regulation of protein phosphatase $2 A$ and its role in controlling entry into and exit from mitosis. Adv Biol Regul 2013; 53: 173-178.

13. Mizuarai S, Yamanaka K, Itadani H, Arai T, Nishibata T, Hirai $\mathrm{H}$ et al. Discovery of gene expression-based pharmacodynamic biomarker for a p53 context-specific anti-tumor drug Wee1 inhibitor. Mol Cancer 2009; 8: 34.

14. D'Angiolella V, Palazzo L, Santarpia C, Costanzo V, Grieco D. Role for non-proteolytic control of M-phase-promoting factor activity at M-phase exit. PLOS One 2007; 2: e247.

15. Morin V, Prieto S, Melines S, Hem S, Rossignol M, Lorca T et al. CDK-dependent potentiation of MPS1 kinase activity is essential to the mitotic checkpoint. Curr Biol 2012; 22: 289-295.

16. Pui CH, Evans WE. Treatment of acute lymphoblastic leukemia. N Engl J Med 2006; 54: 166-178.

17. Do K, Doroshow JH, Kummar S. Wee1 kinase as a target for cancer therapy. Cell Cycle 2013; 12: 3159-3164.

18. Campo E, Swerdlow SH, Harris NL, Pileri S, Stein H, Jaffe ES. The 2008 WHO classification of lymphoid neoplasms and beyond: evolving concepts and practical applications. Blood 2011; 117: 5019-5032.

19. Vitale A, Guarini A, Ariola C, Mancini M, Mecucci C, Cuneo A et al. Adult T-cell acute lymphoblastic leukemia: biologic profile at presentation and correlation with response to induction treatment in patients enrolled in the GIMEMA LAL 0496 protocol. Blood 2006; 107: 473-479.

(c) (i) () $\ominus$ This work is licensed under a Creative Commons Attribution-NonCommercial-NoDerivs 4.0 International License. The images or other third party material in this article are included in the article's Creative Commons license, unless indicated otherwise in the credit line; if the material is not included under the Creative Commons license, users will need to obtain permission from the license holder to reproduce the material. To view a copy of this license, visit http://creativecommons.org/licenses/by-nc-nd/4.0/ 